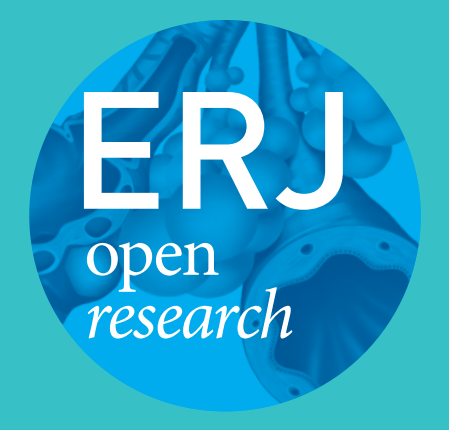

\title{
Determination of the phenotypic age in residents of Mexico City: effect of accelerated ageing on lung function and structure
}

\author{
Ivette Buendía-Roldan ${ }^{1}$, Rosario Fernández-Plata ${ }^{1}$, Abigail Valdes-Bartolo ${ }^{1}$, \\ Mayra Mejia ${ }^{1}$, Luis E. Jaramillo ${ }^{1}$, David Martínez-Briseño ${ }^{1}$, \\ Armando Santiago-Ruiz ${ }^{1}$, Hugo Tapia-Aguilar ${ }^{1}$, Brenda Gómez-Zamora ${ }^{1}$, \\ Annie Pardo ${ }^{2}$ and Moises Selman (10 ${ }^{1}$
}

Affiliations: 'Instituto Nacional de Enfermedades Respiratorias "Ismael Cosío Villegas", México, Mexico. ${ }^{2}$ Facultad de Ciencias, Universidad Nacional Autónoma de México, México, Mexico.

Correspondence: Moises Selman, Instituto Nacional de Enfermedades Respiratorias, Tlalpan 4502; CP 14080 , Mexico City, Mexico. E-mail: mselmanlayahoo.com.mx

\section{ABSTRACT}

Introduction: Phenotypic age better represents age-related biological dysregulation than chronological age. Recently, a multisystem-based ageing measure, which integrates chronological age and nine biomarkers, was proposed.

Methods: Phenotypic age was determined in 774 residents of Mexico City over 60 years old and without respiratory problems. We arbitrarily classified as "accelerated" ageing, those showing $>4$ years compared with their chronological age, and "slowed" ageing, those with $<4$ years compared with chronological age. Demographic risk factors were analysed with structured questionnaires. Lung structure was evaluated by high-resolution computed tomography and functional competence was analysed by forced vital capacity (FVC), forced expiratory volume in $1 \mathrm{~s}\left(\mathrm{FEV}_{1}\right)$, diffusion capacity of carbon monoxide $\left(D_{\mathrm{LCO}}\right)$, and the 6minute walk test (6MWT).

Results: Overall, $13 \%$ of this cohort showed accelerated ageing, which was corroborated with four independent biomarkers of ageing, $42 \%$ had normal ageing and $46 \%$ had slowed ageing. Risk factors associated with accelerated ageing were male sex (OR 4.4, 95\% CI 2.4-7.9; p<0.01), diabetes mellitus (OR 9.7, 95\% CI 5.5-17.2; $\mathrm{p}<0.01$ ), and long-term sleep duration (OR 2.9 95\% CI 1.34-6.35, p<0.01). Among smokers, there was a slight but significant association with the number of pack-years. Subjects with accelerated ageing showed decreased FVC $(p<0.0001), \operatorname{FEV}_{1}(\mathrm{p}<0.0001), D_{\mathrm{LCO}}(\mathrm{p}<0.02)$ and walking distance in the $6 \mathrm{MWT}(\mathrm{p}=0.0001)$. Slowed-ageing subjects presented less frequently with emphysematous lesions compared with those with accelerated ageing.

Conclusions: A small but significant proportion of residents of Mexico City age rapidly, which is associated with male sex, diabetes, and long-term sleep duration. They exhibit lower levels of lung function and develop emphysema more frequently.

@ERSpublications

Systemic accelerated ageing results in decline of lung function and predisposes to emphysema compared with healthy ageing https://bit.ly/3fmeO 82

Cite this article as: Buendía-Roldan I, Fernández-Plata R, Valdes-Bartolo A, et al. Determination of the phenotypic age in residents of Mexico City: effect of accelerated ageing on lung function and structure. ERJ Open Res 2020; 6: 00084-2020 [https://doi.org/10.1183/23120541.00084-2020].

Received: 21 Feb 2020 | Accepted after revision: 30 April 2020

Copyright $\odot$ ERS 2020. This article is open access and distributed under the terms of the Creative Commons Attribution Non-Commercial Licence 4.0. 


\section{Introduction}

The world's population is living longer. The number of people aged 60 years or more worldwide has doubled since 1980 and is estimated to reach 2 billion by 2050 [1]. Ageing is characterised by a progressive decline in homeostasis, which results in an increased risk of disease and death. Lung ageing is a highly complex process, influenced by genetic architecture, lifestyle, environmental factors, and accumulated cellular and molecular changes provoked by multiple injuries and repair [2,3]. In general, normal ageing of the lung is associated with a progressive decrease in its mechanical function, as well as gas exchange and exercise capacity [2]. Regarding structure, some studies suggest that the most frequent alteration of lung ageing is so-called "senile emphysema" characterised by overdistention of the alveolar spaces [4-6]. However, recent studies, with a tiny number of individuals, indicate that in some cases, ageing causes pulmonary function restriction and tomographic lesions suspected of fibrosis [7].

The rate of age-related decline is not universal, and heterogeneity arises due to variations in genetic architecture, exposure to damaging environments, and other factors. Consequently, age, when measured chronologically, may not be a reliable indicator of the body's rate of decline or physiological breakdown [8]. Accordingly, people with the same chronological age can exhibit different ageing rates and therefore show unusual functional deterioration and susceptibility to develop ageing-related diseases $[8,9]$.

In this context, it has been proposed that the phenotypic age better represents the complexity of the ageing process and the age-related biological dysregulation, and facilitates identification of individuals at risk for several chronic diseases [10]. Nevertheless, determination of phenotypic age is very complex and there is no gold standard to estimate an individual's "biological" age. Two algorithms have been proposed based on changes in the methylome $[11,12]$, and more recently, a signature of 513 CpGs (DNAm PhenoAge) was reported to predict age with notable accuracy [13].

However, these analyses are expensive and difficult to perform in clinical practice. Additionally, in longitudinal datasets, it has been shown that chronological age increases at a faster rate than epigenetic estimated age. Likewise, in cross-sectional studies, there are substantial biological sex differences, with men having more significant positive age acceleration than women [14].

A novel multisystem-based ageing measure was recently developed to determine phenotypic age, based on routine biomarkers of clinical chemistry $[15,16]$. This formula has the advantage that it is easy to apply in the clinical setting as the proposed nine biochemical parameters are usually available to any laboratory.

Based on this background, this study aimed to determine the phenotypic age of individuals enrolled in our Ageing Program at the National Institute of Respiratory Diseases (INER), and analyse whether the rate of ageing is related to structural and functional changes of the lung.

\section{Study population and methods}

We are conducting an observational, cross-sectional study, enrolling individuals over 60 years of age, without previous lung diseases and without respiratory symptoms at the time of the study (INER Ageing Program). The data presented here cover a period from March 2015 to April 2019. The project was approved by the INER Ethics and Research Committee (C39-14), and signed informed consent was obtained from all participants. To minimise the effect of chronic exposure to high levels of air pollution, all the enrolled individuals should have lived at least 10 years in Mexico City.

\section{Inclusion criteria:}

1) Age over 60 years.

2) No occupational exposures to toxic materials.

3) Body mass index $>18.5$ and $<30\left(\mathrm{~kg} \cdot \mathrm{m}^{-2}\right)$.

4) Respiratory asymptomatic according to a standard list of symptoms.

\section{Exclusion criteria:}

1) Inability to complete the walking functional tests and spirometry.

2) Subjects with chronic nonrespiratory diseases without medical control (e.g. diabetes mellitus, systemic arterial hypertension, hypothyroidism, epilepsy).

3) Ever treated with chemotherapy or radiotherapy.

\section{Elimination criteria:}

1) Those who wish to withdraw from the study.

\section{Questionnaires}

A modification of the questionnaire used in the PLATINO study (Latin American pulmonary obstruction research project) was applied [17]. We used only the questions that considered demographic data, symptoms and previous respiratory diseases, use of medications for respiratory problems, smoking, 
occupational exposure, comorbidities, economic impact, and exposure to intra-household pollutants. Quality of life was evaluated with the Euro-Qol questionnaire [18].

\section{High-resolution computed tomography of the chest}

The study was carried out in the INER Imaging Service following the protocol for high-resolution computed tomography (HRCT) of the supine position, with sequential acquisition mode, using a 16-slice helical CT (Somatom Sensation 64, software version syngo CT 2014A; Siemens Medical Solutions, Forchheim, Germany). Inspiration and expiration scans were obtained at the end of full inspiration and the end of full expiration. We used the following parameters: $120-140 \mathrm{kVp}, 200 \mathrm{mAs}, 1-\mathrm{mm}$ table feed per rotation, 1-mm collimation, and a $10-\mathrm{mm}$ interval. Image data were reconstructed with $1.0-\mathrm{mm}$ thicknesses and 10-mm intervals.

We examined the presence of different structural alterations, such as interstitial lung abnormalities defined as the occurrence of ground-glass opacities, reticular abnormalities, diffuse centrilobular nodules, honeycombing, traction bronchiectasis, nonemphysematous cysts or architectural distortion involving at least $5 \%$ of nondependent portions of the lung [19]. Emphysema was defined on inspiratory CT scans as the presence of decreased attenuation areas (less than $-910 \mathrm{HU}$ ) with a decrease in diameter and number of pulmonary vessels in the area of the emphysema [20]. Evidence of air-trapping was examined in the expiratory phase and characterised by the presence of patchy areas of low attenuation density and lack of lung volume reduction [21].

\section{Spirometry}

Spirometry was carried out with the Easy One Pro-Lab equipment. Three repeatable tests (with a difference of $<150 \mathrm{~mL}$ between them) were obtained to assess the best forced vital capacity (FVC), forced expiratory volume in $1 \mathrm{~s}\left(\mathrm{FEV}_{1}\right)$ and the $\mathrm{FEV}_{1} / \mathrm{FVC}$ ratio. Spirometry was performed without bronchodilators.

\section{Diffusing capacity of the lung for carbon monoxide}

A study of the diffusing capacity of the lung for carbon monoxide $\left(D_{\mathrm{LCO}}\right)$ was carried out with the Easy One Pro-Lab ${ }^{\oplus}$ equipment, obtaining three repeatable tests to record the best value in $\mathrm{mL} \cdot \mathrm{min}^{-1} \cdot \mathrm{mmHg}^{-1}$ and percentage.

\section{6-minute walk test}

The 6-minute walk test (6MWT) was performed after spirometry and lung diffusion with a previous rest of $10 \mathrm{~min}$. Resting oxygen saturation was recorded with a NONIN ${ }^{\circ}$ pulse oximeter and blood pressure. Subsequently, the walk was carried out for $6 \mathrm{~min}$ in a 20 -metre aisle circuit, recording at the end the number of metres walked as well as oxygen saturation, blood pressure and BORG scale (from 0 to 10) to assess dyspnoea and fatigue.

\section{Biomarkers to determine phenotypic age}

Nine variables were evaluated: albumin, creatinine, glucose, C-reactive protein, absolute number of leukocytes and percentage of circulating lymphocytes, mean corpuscular volume, erythrocyte distribution range and alkaline phosphatase were measured in the clinical laboratory using standard methods.

Phenotypic age was calculated according to the following formula $[15,16]$ :

$$
\text { Phenotypic Age }=141.50+\frac{\ln (-0.00553 \times \ln (1-\mathrm{xb})}{0.09165}
$$

where $\mathrm{xb}$ is constructed with a linear combination of chronological age and the nine mentioned biomarkers.

We arbitrarily classify individuals with "accelerated" ageing as those who had more than 4 years of their chronological age, with "normal" ageing as those with a difference of 0 to 4 years of their chronological age, and with "slowed" ageing as those who had less than 4 years of their chronological age.

\section{Statistical analysis}

Descriptive data were analysed as proportions, or mean and SD. Group comparisons were performed by using a t-test or ANOVA for interval variables, and Pearson's Chi-squared or Fisher's exact test for categorical variables. In the case of multiple comparisons, the Bonferroni correction was applied. Spearman correlation was used to assess the association between baseline FVC\% and phenotypic age. A p-value $<0.05$ was considered statistically significant. A multinomial logistic regression model was used to 
estimate odds ratio (OR) and 95\% confidence interval (CI) with Stata version 15.0 (StataCorp, College Station, TX, USA) and R program version 3.5.3 (St Louis, MO, USA).

\section{Results}

\section{Determination of phenotypic age}

We evaluated 774 subjects from the cohort of the Ageing Program at INER to determine their phenotypic ages and lung consequences (figure 1). All of them were residents of Mexico City and had been for at least the last 10 years to minimise the effect of altitude and air pollution.

As illustrated in figure 2, our results revealed that 13\% (99 of 774) of the participants in this cohort showed accelerated ageing ( $11 \pm 6.9$ years above their chronological age), 42\% (322 of 774 ) showed normal ageing, and $46 \%$ (353 of 774) showed slowed ageing ( $7.4 \pm 2.3$ years less than chronological age).

Accelerating ageing was further corroborated with independent measurement of other parameters associated with ageing, such as apolipoprotein A, triglycerides, body mass index, and glycosylated haemoglobin (HbA1c) (figure 3). Since HbA1c increases with diabetes that was markedly increased in the accelerated group (table 1), we also examined HbA1c levels, excluding patients with diabetes. HbA1c levels remained significantly elevated in subjects with accelerated ageing $(6.8 \pm 4.3 \%$ versus $5.7 \pm 0.8 \%, \mathrm{p}<0.0001)$ supporting the notion that HbAlc shows a steady increase with age, and is positively associated with ageing in nondiabetic populations [22].

The comparison of clinical and demographic variables analysed among the three groups showed that accelerated ageing was associated with male sex, diabetes mellitus, and long-term sleep duration (more than $8 \mathrm{~h} \cdot \mathrm{day}^{-1}$ ) (table 1). They also displayed lower Euro-Qol scores in parameters related to mobility. Slowed-ageing subjects displayed a lower frequency of systemic arterial hypertension (table 1). No association was found with other comorbidities usually associated with ageing such as gastro-oesophageal reflux.

Comparing the group of subjects with accelerated ageing versus those with slowed ageing using multinomial logistic regression models, we found that male sex (OR 4.4, 95\% CI 2.5-7.9; $\mathrm{p}<0.01$ ), diabetes mellitus (OR 9.7, 95\% CI 5.56-17.2; p<0.01), and long-term sleep duration (OR 2.9, 95\% CI 1.34-6.35; $\mathrm{p}<0.01)$ were associated with accelerated ageing. Although no association was observed with smoking, we found that among smokers, there was a slight but significant association of accelerated ageing with the number of pack-years (OR=1.02, 95\% CI 1.001-1.04; $\mathrm{p}=0.04)$.

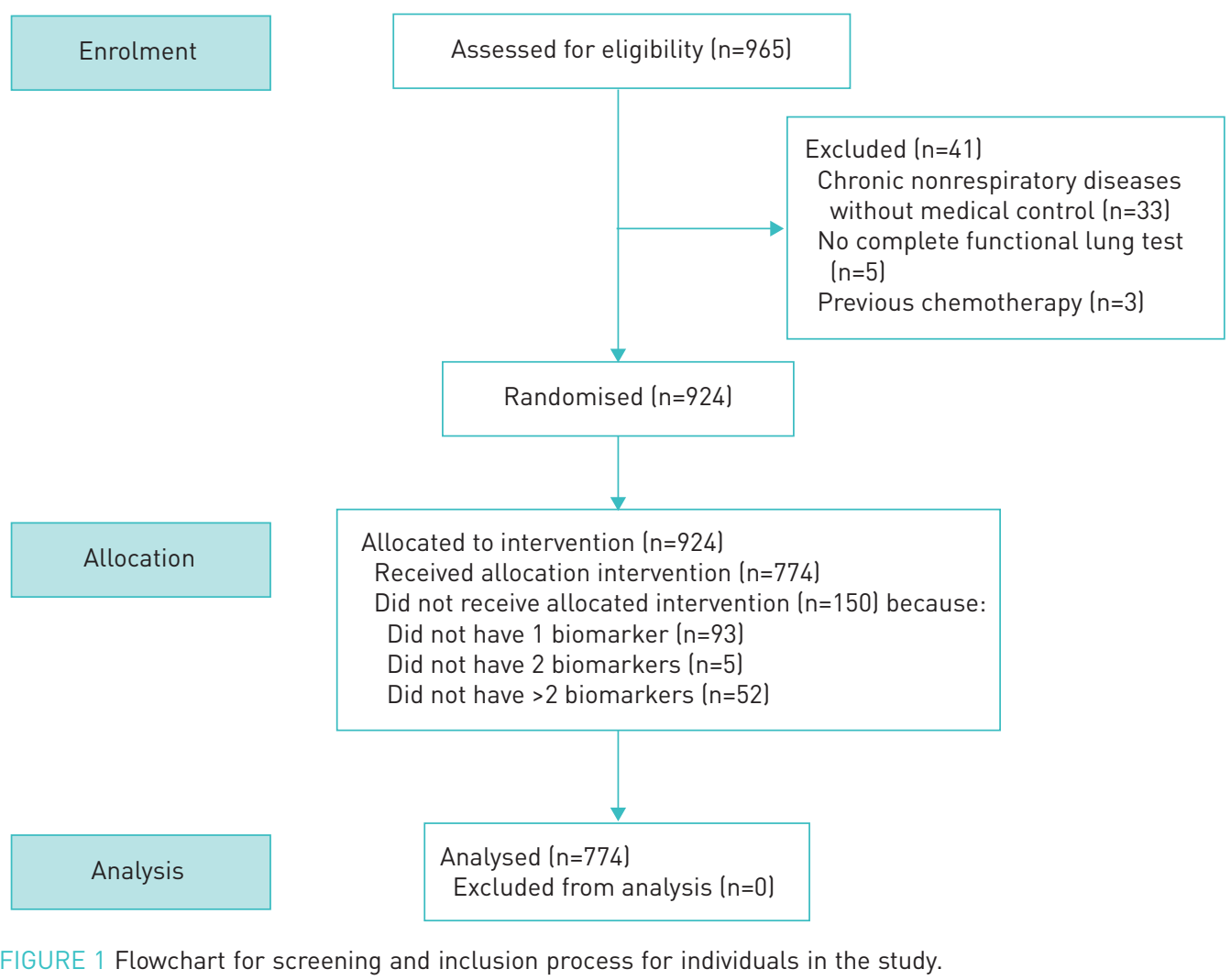


FIGURE 2 Box-and-whisker diagram of chronological and phenotypic age. Three quartiles and the minimum and maximum values of the chronological and phenotypic age are shown.

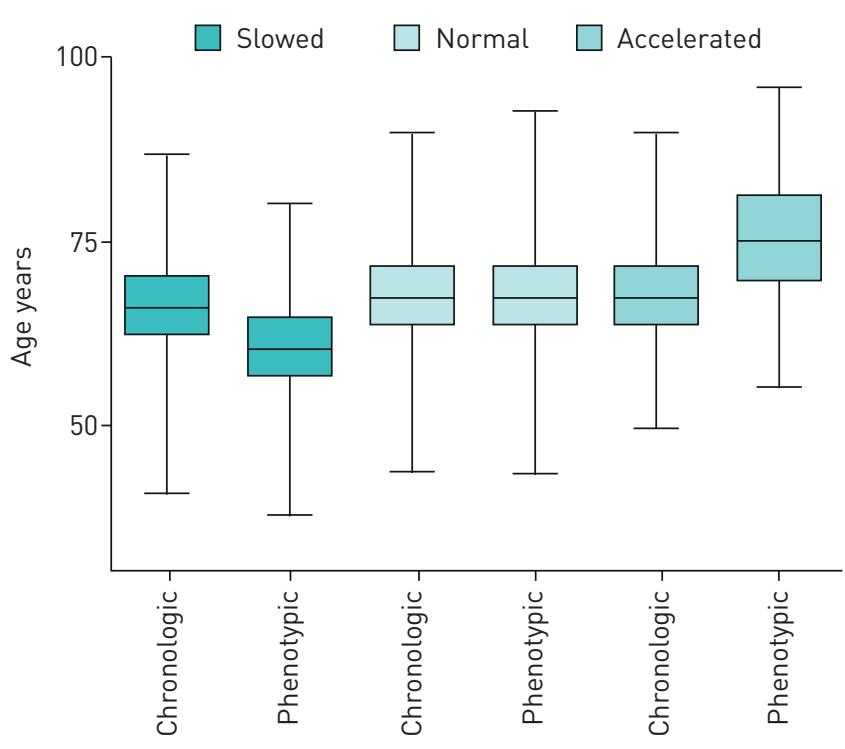

\section{Pulmonary function tests and phenotypic age}

When the respiratory function was compared among individuals with accelerated, normal, and slowed ageing, a significant decrease in FVC $(\mathrm{p}<0.0001), \mathrm{FEV}_{1}(\mathrm{p}<0.0001), D_{\mathrm{LCO}}(\mathrm{p}=0.02), D_{\mathrm{LCO}}$ /alveolar volume $\left(V_{\mathrm{A}}\right)(\mathrm{p}=0.03)$, and walking distance in the 6MWT $(\mathrm{p}=0.0001)$ were observed in the accelerated ageing group, as compared with the other two groups. The accelerated ageing group also showed a reduction in resting oxygen saturation $(\mathrm{p}<0.05)($ table 2$)$.

A statistically significant negative correlation was found between phenotypic age and FVC ( $\mathrm{r}=-0.17$, $\mathrm{p}<0.0001$, figure 4), indicating that FVC decreased as phenotypic age advanced.

Table 3 shows the pairwise statistical differences in clinical and functional variables after adjustment by Bonferroni correction. As can be seen in this table, the majority of statistically significant differences were
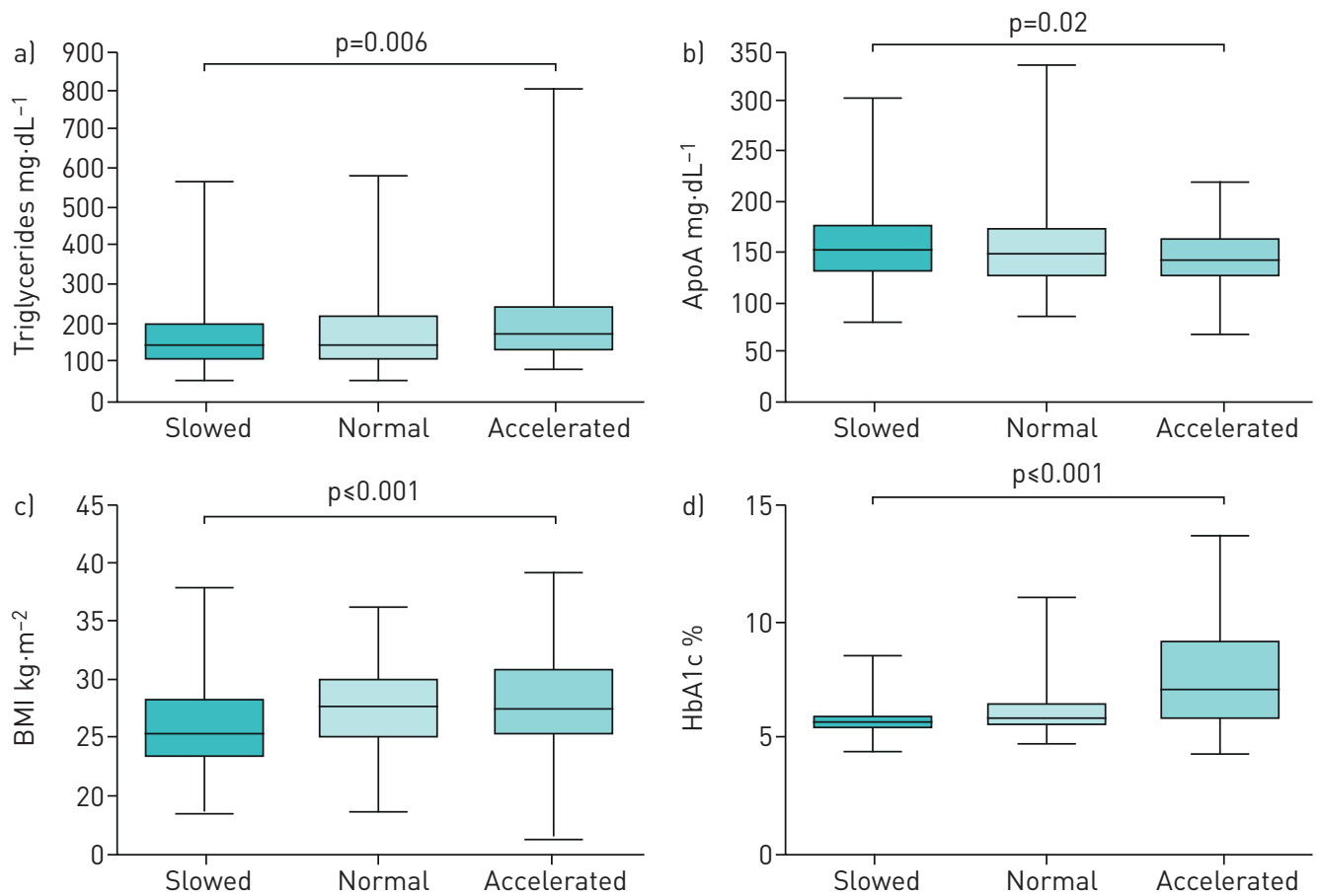

FIGURE 3 Box-and-whisker diagram of independent measurement of parameters associated with ageing. One clinical and three laboratory parameters show significant differences among the three groups by one-way ANOVA. BMI: body mass index; HbA1c: glycosylated haemoglobin; ApoA: apolipoprotein A. 


\begin{tabular}{|c|c|c|c|c|}
\hline & $\begin{array}{c}\text { Slowed ageing } \\
n=353\end{array}$ & $\begin{array}{c}\text { Normal ageing } \\
n=322\end{array}$ & $\begin{array}{l}\begin{array}{c}\text { Accelerated ageing } \\
\mathrm{n}=99\end{array}\end{array}$ & p-value \\
\hline \multicolumn{5}{|l|}{ Variable } \\
\hline Chronological age years $^{+}$ & $68 \pm 7$ & $68 \pm 7$ & $70 \pm 8$ & 0.0005 \\
\hline Sex, male $\#$ & $61(17 \%)$ & $135(42 \%)$ & $42(42 \%)$ & $<0.0001$ \\
\hline Smoking history" & $161(46 \%)$ & $168(52 \%)$ & $52(53 \%)$ & NS \\
\hline Diabetes mellitus ${ }^{\#}$ & $41(12 \%)$ & $73(23 \%)$ & $51(52 \%)$ & $<0.0001$ \\
\hline Systemic arterial hypertension ${ }^{\#}$ & $125(36 \%)$ & $142(44 \%)$ & $44(45 \%)$ & 0.05 \\
\hline Body mass index $\mathrm{kg} \cdot \mathrm{m}^{+}$ & $26 \pm 3.7$ & $28 \pm 4.3$ & $28 \pm 4.3$ & $<0.0001$ \\
\hline Long-sleep duration\#" & $29(8 \%)$ & $22(7 \%)$ & $17(17 \%)$ & 0.003 \\
\hline \multicolumn{5}{|l|}{ Quality of life (EuroQol) } \\
\hline Problems with walking" & $30(9 \%)$ & $32(10 \%)$ & $15(15 \%)$ & 0.05 \\
\hline Problems with dressing ${ }^{\Uparrow}$ & $4(1 \%)$ & $11(3 \%)$ & $6(6 \%)$ & 0.008 \\
\hline Problems with daily activities & $12(3 \%)$ & $14(4 \%)$ & $9(9 \%)$ & 0.03 \\
\hline
\end{tabular}

found when slowed and accelerated groups were compared, although some significant differences were also observed when slowed and accelerated ageing groups were independently compared with the normal group.

\section{Structural lung changes and phenotypic age}

Lung structure was examined with HRCT scans. Image analysis showed that $42 \%$ of tomographic scans were completely normal. In 5\% of the individuals, HRCT was normal during inspiration, but they showed different degrees of air-trapping in the expiratory phase, which represents the premature closure of peripheral airways usually associated with loss of pulmonary elasticity; the remaining 53\%, displayed various (typically mild) structural alterations including emphysema, bronchiectasis, and interstitial pulmonary abnormalities suggestive of inflammation and/or fibrosis.

Analysis of these structural alterations in different ageing phenotypes showed that individuals with slowed ageing presented a significantly lower frequency of pulmonary emphysema compared with accelerated and normal-ageing subjects $(\mathrm{p}<0.0001)$ (table 2, fig. 5). None of the other structural alterations was associated with the type of phenotypic ageing.

\section{Discussion}

There is strong evidence that people have essentially two ages, the chronological, which is given by the calendar according to the date of birth, and the phenotypic (or biological), which represents the "real" functional state of the organism. In this context, people who have a phenotypic age higher than their

TABLE 2 Baseline pulmonary function tests and emphysematous changes in the three study groups

Slowed ageing $n=353$ Normal ageing $n=322$ Accelerated ageing $n=99 \quad p$-value

$\begin{array}{lcccc}\text { Variable } & & & \\ \text { FVC \% pred } & 97 \pm 15 & 93 \pm 15 & 89 \pm 17 & <0.0001 \\ \text { FEV } \% \text { pred } & 102 \pm 16 & 98 \pm 17 & 93 \pm 19 & <0.0001 \\ D_{\text {LCO }} \text { adjusted } & 99 \pm 18 & 99 \pm 19 & 91 \pm 24 & 0.02 \\ D_{\text {LCo }} / V_{\text {A }} & 106 \pm 19 & 108 \pm 21 & 101 \pm 22 & 0.03 \\ \text { 6MWT m } & 455 \pm 122 & 454 \pm 114 & 396 \pm 152 & 0.0001 \\ \mathrm{O}_{2} \text { saturation at rest } & 94.3 \pm 2.1 & 94.3 \pm 1.9 & 93.9 \pm 2.1 & <0.05 \\ \text { Emphysema } & 13(4 \%) & 26(8 \%) & 8(9 \%) & <0.0001\end{array}$

Data are presented as mean \pm SD or $n(\%)$, unless otherwise stated. FVC: forced vital capacity; \% pred $\%$ predicted; $\mathrm{FEV}_{1}$ : forced expiratory volume in $1 \mathrm{~s} ; D_{\mathrm{Lco}}$ : diffusing capacity carbon monoxide; $V_{\mathrm{A}}$ : alveolar volume; 6MWT: 6-min walking test. 
FIGURE 4 Scatter plot showing a negative correlation between forced vital capacity (FVC) \% predicted and phenotypic age in the whole cohort.

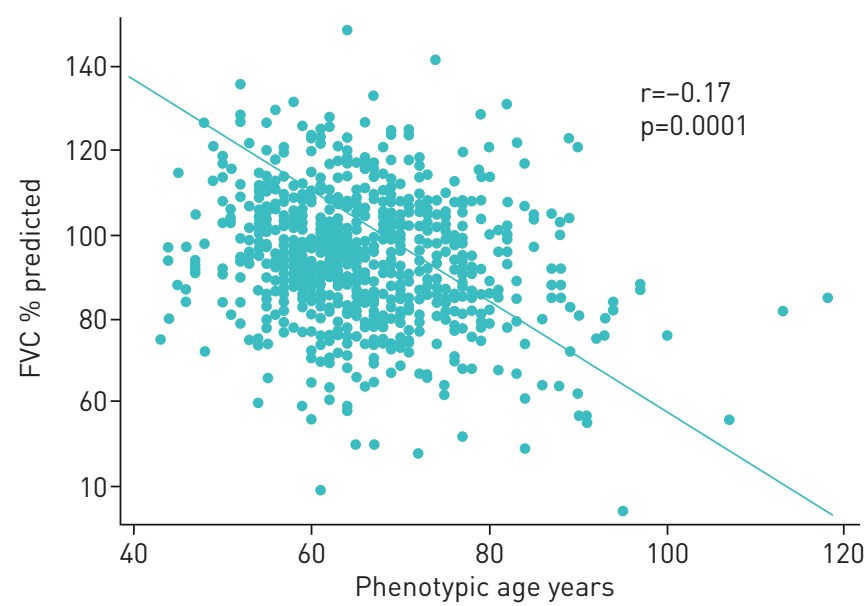

chronological age (here called accelerated ageing) have substantial functional impairment and risk of developing ageing-associated diseases than those whose phenotypic age is less than the chronological age (which we call here, slowed ageing) $[9,15]$.

In this context, numerous studies are indicating that ageing contributes to pathogenesis of several chronic lung diseases, some of them virtually exclusive to older adults, whereas others may occur at any age, usually with a worse prognosis in adults over 65 years [23]. However, these studies have been carried out considering chronological age, which offers limited information regarding the complex processes driving biological ageing $[9,24]$. Thus, it is the phenotypic age, usually influenced by a complex combination of genetic, lifestyle, and environmental factors that plays an important role in susceptibility to morbidity and mortality [10, 25]. In this context, studies of respiratory function and pathology considering the phenotypic age are scanty.

In this study, we applied a recently proposed method to determine phenotypic age to an ongoing cohort of our Ageing Program. The equation has the advantage that the components cover different organs, are minimally invasive, and reliably measured [15]. Interestingly, we found that $13 \%$ of older adults who entered the study, all residents of Mexico City, showed accelerated ageing ( $>4$ years of chronological age).

The comparison of the accelerated group with normal and slowed groups demonstrated that subjects with accelerated ageing were mainly males and showed a higher frequency of diabetes. Also, although it was observed in a few subjects, those with accelerated ageing showed a higher incidence of mobility problems, which affects health-related quality of life.

Using multinomial logistic regression models, we found that male sex and diabetes markedly increase the risk of accelerated ageing. The stronger finding with diabetes supports previous studies that have

\section{TABLE 3 Differences of clinical and functional variables between groups ${ }^{\#}$}

Variable

Chronological age years

Body mass index $\mathrm{kg} \cdot \mathrm{m}^{-2}$

\section{Laboratory}

Glycosylated haemoglobin \%

Apolipoprotein $\mathrm{A} \mathrm{mg} \cdot \mathrm{dL}^{-1}$

Triglycerides $\mathrm{mg} \cdot \mathrm{dL}^{-1}$

Lung function tests

FVC \% pred

$\mathrm{FEV}_{1} \%$ pred

$D_{\text {LCo }}$ adjusted

$D_{\mathrm{LCO}} / V_{\mathrm{A}}$

6MWT m

$\mathrm{O}_{2}$ saturation at rest
Normal versus slowed

0.65
$1.67 *$
$0.39 *$
-1.35
11.31
$-4.60^{*}$
$-434^{*}$
0.31
1.76
-2.08
-0.02

Accelerated versus slowed

$2.48 *$

$1.97 *$

2.05*

$-10.52^{*}$

44.0*

$-8.44^{*}$

$-8.66 *$

$-7.72 *$

$-4.90$

$-56.22 *$

$-067 *$

Accelerated versus normal

1.83

0.29

$1.67 *$

$-9.16^{*}$

$32.69 *$

$-3.84$

$-4.32$

$-8.03^{*}$

$-6.66 *$

$-54.14^{*}$

$-0.65^{*}$

\#: One-way ANOVA was performed using Bonferroni method for multiple comparisons. FVC: forced vital capacity; FEV $_{1}$ : forced expiratory volume in $1 \mathrm{~s}$; $D_{\mathrm{LCO}}$ : diffusing capacity carbon monoxide; $V_{\mathrm{A}}$ : alveolar volume; 6MWT: 6-min walking test. *: $p<0.05$. 
FIGURE 5 Structural changes associated with accelerated ageing. Emphysematous lesions (red arrows) in a male smoker with a chronological age of 68 years and calculated phenotypic age of 73 years.

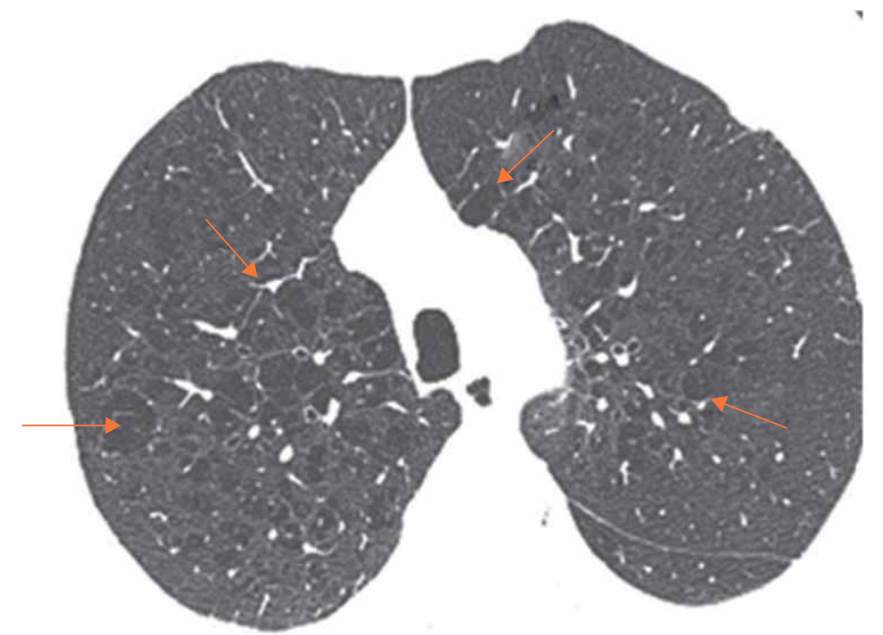

demonstrated that this disease, together with obesity, is associated with accelerated ageing. Thus, for example, a recent study using three models to determine phenotypic age showed that people with diabetes and systemic hypertension were phenotypically older than their slowed controls matched by chronological age [10]. Regarding systemic hypertension, we found that individuals with slowed ageing have a lower frequency of this disease, but no differences were noted between accelerated and normal-ageing groups. Also, although we do not found differences between smokers and nonsmokers, among smokers, the individuals that smoked a higher number of pack-years showed increased phenotypical age. There is evidence that smoking, especially the volume of smoked cigarettes, is associated with accelerated ageing, which is probably related to an effect of the components of tobacco smoke on telomere shortening [26, 27]. Interestingly, long-term sleep duration was a risk factor for having an accelerated phenotype. In this context, a growing body of evidence indicates that long and short sleep duration increases the risk of developing or dying of several ageing-associated diseases, such as coronary heart disease and stroke [28], type 2 diabetes [29] and cancer [30].

On the other hand, the effect of accelerated ageing on lung structure and function is uncertain. In this study, which included older adults without significant prior lung diseases nor respiratory symptoms, accelerated ageing was associated with a substantial decrease in lung function, compared to those showing normal or slowed ageing. The impairment detected in accelerated-age individuals included mechanical function (FVC and $\left.\mathrm{FEV}_{1}\right)$ and gas exchange capacity $\left(D_{\mathrm{LCO}}, \mathrm{O}_{2}\right.$ saturation at rest, and walking distance in the $6 \mathrm{MWT}$ ). Similar results were very recently reported in a cross-sectional analysis for both baseline and follow-up time points, where accelerated ageing was determined by peripheral blood epigenetic signature [31]. This study, however, evaluated a younger population and included patients with asthma. In addition, lung diffusion capacity and lung structural changes by HRCT were not examined.

Structural evaluation of the lungs in our cohort showed that the frequency of pulmonary emphysema was markedly lower in individuals with slowed ageing compared with both normal and accelerated ageing groups, suggesting a protective role. Importantly and perhaps surprisingly, no differences were noticed between subjects with normal and accelerated ageing. Pulmonary emphysema is a chronic, progressive, and irreversible disease characterised by the destruction of the alveolar walls usually associated with tobacco smoking and chronological age [32, 33].

Our study has some limitations. The sample size is relatively small, it has been developed so far only in Mexico City (with high air pollution), and the questionnaires did not include some questions about factors that can contribute to accelerated ageing, particularly those related to stress during childhood. Likewise, our questionnaires asked specifically on the use of medications to respiratory problems but did not explore in detail the use of other medications throughout life. Also, Levine's score used in this study has relatively limited testing variables, and since it was recently proposed, lack replication and validation studies in different clinical settings. In this context, unexpectedly, we found a high percentage of our cohort displaying slowed ageing. The reason for this finding is unknown, but it may represent a particular healthy set of individuals or maybe a bias of Levine's algorithm.

Importantly, however, our results regarding accelerated ageing were corroborated with four independent biomarkers of ageing and, as mentioned, similar results were reported using epigenetic age acceleration with levels of FVC and $\mathrm{FEV}_{1}$, mainly in women older than 50 years [31]. 
Moreover, the distribution of pulmonary structural changes associated with ageing that we find in our cohort is very similar to what is reported in other cohorts with different genetic backgrounds, living at sea level, and in less polluted areas. Thus, for example, we have found the presence of interstitial lung abnormalities, bronchiectasis, loss of elasticity of the peripheral airways and air-trapping equivalent to that published in other populations [34-37].

In summary, our findings suggest that a small but significant proportion of the population living in Mexico City ages rapidly and that these individuals show a reduction in their functional respiratory capacity. Our results also show that people displaying a slower ageing rate present a lower risk of developing pulmonary emphysema.

Author contributions: I. Buendía-Roldan, M. Selman and A. Pardo conceived the study. A. Valdes-Bartolo, L.E. Jaramillo and A. Santiago-Ruiz performed the literature search. H. Tapia-Aguilar and B. Gómez-Zamora collected the data. R. Fernández-Plata, D. Martínez-Briseño and M. Mejia were responsible for the methodology. I. Buendía-Roldan, R. Fernández-Plata, A. Valdes-Bartolo and M. Selman performed the formal analysis. M. Selman wrote the original draft of the manuscript. Ivette Buendía-Roldan, Annie Pardo, Rosario Fernández-Plata wrote, reviewed and edited the final manuscript.

Conflict of interest: None declared.

Support statement: This study was supported by "Secretaría de Educación, Ciencia, Tecnología e Innovación de la Ciudad de México" (grant number SECITI/115/2017) and CONACYT (grant number FOSISS 290645). Funding information for this article has been deposited with the Crossref Funder Registry.

\section{References}

1 United Nations. The 2019 Revision of World Population Prospects. United Nations/Population Division. World Population Prospects 2019. http://esa.un.org/unpd/wpp Date last accessed: November 2019. Date last updated: 28 August 2019.

2 Cho SJ, Stout-Delgado HW. Aging and lung disease. Ann Rev Physiol 2020; 82: 16.1-16.27.

3 Fragoso CA V, Gill TM. Respiratory impairment and the aging lung: a novel paradigm for assessing pulmonary function. J Gerontol A Biol Sci Med Sci 2012; 67: 264-275.

4 Verbeken EK, Cauberghs M, Lauweryns JM, et al. Anatomy of membranous bronchioles in normal, senile, and emphysematous human lungs. J Appl Physiol (1985) 1994; 77: 1875-1884.

5 Hochhegger B, Alves GR, Irion KL, et al. Emphysema index in a cohort of patients with no recognizable lung disease: influence of age. J Bras Pneumol 2012; 38: 494-502.

6 Janssens JP, Pache JC, Nicod LP. Physiological changes in respiratory function associated with aging. Eur Respir J 1999; 13: 197-205.

7 Copley SJ, Wells AU, Hawtin KE, et al. Lung morphology in the elderly: comparative CT study of subjects over 75 years old versus those under 55 years old. Radiology 2009; 251: 566-573.

8 Levine ME. Modeling the rate of senescence: can estimate biological age predict mortality more accurately than chronological age? J Gerontol A Biol Sci Med Sci 2013; 68: 667-674.

9 Khan SS, Singer BD, Vaughan DE. Molecular and physiological manifestations and measurement of aging in humans. Aging Cell 2017; 16: 624-633.

10 Liu Z, Chen X, Gill TM, et al. Associations of genetics, behaviors, and life course circumstances with a novel aging and healthspan measure: Evidence from the Health and Retirement Study. PLoS Med 2019; 16: e1002827.

11 Horvath S. DNA methylation age of human tissues and cell types. Genome Biol 2013; 14: R115.

12 Hannum G, Guinney J, Zhao L, et al. Genome-wide methylation profiles reveal quantitative views of human aging rates. Mol Cell 2013; 49: 359-367.

13 Levine ME, Lu AT, Quach A, et al. An epigenetic biomarker of aging for lifespan and healthspan. Aging (Albany, NY) 2018; 10: 573-591.

14 Bell CG, Lowe R, Adams PD, et al. DNA methylation aging clocks: challenges and recommendations. Genome Biol 2019; 20: 249 .

15 Liu Z, Kuo p-L, Horvath S, et al. A new aging measure captures morbidity and mortality risk across diverse subpopulations from NHANES IV: a cohort study. PLoS Med 2018; 15: e1002718.

16 Liu Z, Kuo PL, Horvath S, et al. Correction: a new aging measure captures morbidity and mortality risk across diverse subpopulations from NHANES IV: a cohort study. PLoS Med 2019; 16: e1002760.

17 Menezes AM, Perez-Padilla R, Jardim JR, et al. Chronic obstructive pulmonary disease in five Latin American cities (the PLATINO study): a prevalence study. Lancet 2005; 366: 1875-1881.

18 Herdman M, Gudex C, Lloyd A, et al. Development and preliminary testing of the new five-level version of EQ-5D (EQ-5D-5L). Qual Life Res 2011; 20: 1727-1736.

19 Washko GR, Hunninghake GM, Fernandez IE, et al. Lung volumes and emphysema in smokers with interstitial lung abnormalities. N Engl J Med 2011; 364: 897-906.

20 Goddard PR, Nicholson EM, Laszlo G, et al. Computed tomography in pulmonary emphysema. Clin Radiol 1982; 33: 379-387.

21 Hansell DM, Bankier AA, MacMahon H, et al. Fleischner Society: glossary of terms for thoracic imaging. Radiology 2008; 246: 697-722.

22 Pani LN, Korenda L, Meigs JB, et al. Effect of aging on A1C levels in individuals without diabetes: evidence from the Framingham Offspring Study and the National Health and Nutrition Examination Survey 2001-2004. Diabetes Care 2008; 31: 1991-1996.

23 Thannickal VJ, Murthy M, Balch WE, et al. Blue journal conference. Aging and susceptibility to lung disease. Am J Respir Crit Care Med 2015; 191: 261-269. 
24 Pyrkov TV, Slipensky K, Barg M, et al. Extracting biological age from biomedical data via deep learning: too much of a good thing? Sci Rep 2018; 8: 5210.

25 Belsky DW, Moffitt TE, Cohen AA, et al. Eleven telomere, epigenetic clock, and biomarker-composite quantifications of biological aging: do they measure the same thing? Am J Epidemiol 2018; 187: 1220-1230

Fiorito G, McCrory C, Robinson O, et al. Socioeconomic position, lifestyle habits, and biomarkers of epigenetic aging: a multi-cohort analysis. Aging (Albany NY) 2019; 11: 2045-2070.

27 Müezzinler A, Mons U, Dieffenbach AK, et al. Smoking habits and leukocyte telomere length dynamics among older adults: results from the ESTHER cohort. Exp Gerontol 2015; 70: 18-25.

28 Cappuccio FP, Cooper D, D’Elia L, et al. Sleep duration predicts cardiovascular outcomes: a systematic review and meta-analysis of prospective studies. Eur Heart J 2011; 32: 1484-1492.

29 Yadav D, Cho KH. Total sleep duration and risk of type 2 diabetes: evidence-based on clinical and epidemiological studies. Curr Drug Metab 2018; 19: 979-985.

30 Chen Y, Tan F, Wei L, et al. Sleep duration and the risk of cancer: a systematic review and meta-analysis, including dose-response relationship. BMC Cancer 2018; 18: 1149.

31 Rezwan FI, Imboden M, Amaral AFS, et al. Association of adult lung function with accelerated biological aging. Aging (Albany NY) 2020; 12: 518-542.

32 Celli BR, MacNee W, ATS/ERS Task Force. Standards for the diagnosis and treatment of patients with COPD: a summary of the ATS/ERS position paper. Eur Respir J 2004; 23: 932-946.

33 Brandsma CA, Van den Berge M, Hackett TL, et al. Recent advances in chronic obstructive pulmonary disease pathogenesis: from disease mechanisms to precision medicine. J Pathol 2019; 250: 624-635.

34 Putman RK, Gudmundsson G, Araki T, et al. The MUC5B promoter polymorphism is associated with specific interstitial lung abnormality subtypes. Eur Respir J 2017; 50: 1700537.

35 Quinn TM, Hill AT. Non-cystic fibrosis bronchiectasis in the elderly: current perspectives. Clin Interv Aging 2018; 13: 1649-1656.

36 Lee KW, Chung SY, Yang I, et al. Correlation of aging and smoking with air trapping at thin-section CT of the lung in asymptomatic subjects. Radiology 2000; 214: 831-836.

37 Fukuchi Y. The aging lung and chronic obstructive pulmonary disease: similarity and difference. Proc Am Thorac Soc 2009; 6: 570-572. 